\title{
An SU-8-based microprobe with a nanostructured surface enhances neuronal cell attachment and growth
}

\author{
Eunhee Kim ${ }^{1,2}$, Jin-Young Kim ${ }^{1,2}$ and Hongsoo Choi ${ }^{1,2^{*}}$ (1)
}

\begin{abstract}
Microprobes are used to repair neuronal injury by recording electrical signals from neuronal cells around the surface of the device. Following implantation into the brain, the immune response results in formation of scar tissue around the microprobe. However, neurons must be in close proximity to the microprobe to enable signal recording. A common reason for failure of microprobes is impaired signal recording due to scar tissue, which is not related to the microprobe itself. Therefore, the device-cell interface must be improved to increase the number of neurons in contact with the surface. In this study, we developed nanostructured SU-8 microprobes to support neuronal growth. Nanostructures of $200 \mathrm{~nm}$ diameter and depth were applied to the surface of microprobes, and the attachment and neurite outgrowth of PC12 cells on the microprobes were evaluated. Neuronal attachment and neurite outgrowth on the nanostructured microprobes were significantly greater than those on non-nanostructured microprobes. The enhanced neuronal attachment and neurite outgrowth on the nanostructured microprobes occurred in the absence of an adhesive coating, such as poly-L-lysine, and so may be useful for implantable devices for long-term use. Therefore, nanostructured microprobes can be implanted without adhesive coating, which can cause problems in vivo over the long term.
\end{abstract}

Keywords: Surface modification, Nanostructured surface, Nanosphere lithography, Cell attachment, Neurite outgrowth

\section{Background}

Microprobes are used to repair or bypass brain injury by acquiring electrical signals from neuronal cells in contact with their surface. For reliable neural recording, a stable and sustainable interface between neuronal cells and the microprobes is required. A critical issue for microprobes is the difficulty in maintaining their recording performance for long periods [1], which is due to the biological response of the surrounding cells [2]. Therefore, means of improving the lifetime and biocompatibility of implanted microprobes have been investigated [3].

Neural recording can be improved by applying a bioactive coating (such as of a protein or polymer) to the

\footnotetext{
*Correspondence: mems@dgist.ac.kr

1 DGIST-ETH Microrobotics Research Center (DEMRC), Daegu Gyeongbuk Institute of Science and Technology (DGIST), Daegu 42988, South Korea Full list of author information is available at the end of the article
}

microprobe surface to enhance neuronal cell attachment. For example, Bellamkonda and colleagues used a thin coating of polyethyleneimine (PEI)-laminin (LN) to control scar tissue formation around implanted Si-based neural probes [4]. The PEI-LN-coated silicon microelectrode arrays showed less gliosis at 4 weeks after implantation $[4,5]$. However, attachment of neuronal cells to the microelectrode surface was not enhanced. A polyethylene glycol (PEG) coating containing L1 protein, which is a neural adhesion molecule, increased neurite growth and reduced glial adhesion eightfold compared to a laminin coating [6]. A brain-derived neurotrophic factor (BDNF)-loaded hydrogel coating on planar microelectrode arrays (MEAs) affected neuron process growth and spontaneous electrical activity compared with control MEAs [7]. However, the duration of release is dependent on the amount of protein loaded and the bioactive molecules in the coating; that is, it is limited. Therefore, 
further research is required to enhance our understanding of the microprobe-neuron interaction to prolong recording of neuronal signals.

Rough surfaces, especially nanostructured surfaces, maintain neuron viability more effectively than smooth surfaces, presumably because the former mimic the extracellular matrix $[8,9]$. Various nanostructured surfaces-such as nanowire arrays $[10,11]$, nanopatterned poly- $\varepsilon$-caprolactone (PCL) [12], and a nanoporous gold (np-Au) substrate [13]-increased neuronal growth compared with that on a flat surface. The morphology of np-Au selectively reduces astrocyte surface coverage while maintaining neuronal surface coverage, similar to unstructured planar gold surfaces [14]. Ceramic-based microelectrodes using nanostructured porous silicon were proposed for use as a neural probe [15]. Neurons preferred the nanostructured surface and extended a significantly greater number of neurites, while glial cells avoided the nanostructured surfaces. Subsequent in vivo studies showed that microelectrodes of nanostructured porous silicon could be used to record signals from single neurons [16]; at 1 week, glial activation was reduced and a greater number of neurons were adjacent to the porous silicon surfaces than to the smooth silicon surfaces. Microprobes made of soft materials as an alternative to silicon $(\mathrm{Si})$ and ceramic-based neural probes have been developed and can suppress the inflammatory response after implantation. Although neuronal cells prefer a nanostructured surface, the majority of flexible microprobes have a flat surface. In our previous work, a nanostructured SU-8 substrate exhibited similar neuronal outgrowth to a flat SU-8 surface coated with poly-L-lysine (PLL), which facilitates adhesion by rat pheochromocytoma (PC12) cells $[17,18]$. These findings motivated us to develop SU-8-based microprobes with nanostructures to support neuronal cells on their surface over a long period of time.

Here we present a method for the preparation of nanostructured SU-8 microprobes to improve neuronal binding and growth. We used SU-8 because of its biocompatibility and suitability for neural interface implants $[19,20]$. We can apply the proposed procedure to the preparation of planar microprobes, as discussed in our previous work [17]. Therefore, the microprobes were prepared by conventional photolithography and the nanostructures on their surface were then realized by nanospheric lithography (NSL) [21, 22]. In addition, a nanostructured surface can be patterned as desired simply by covering undesired areas with a thin layer of metal. Scanning Electron Microscopy (SEM) and Atomic Force Microscopy (AFM) were used to characterize the surface nanostructures. The adhesion and outgrowth of PC12 cells was improved on the nanostructured SU-8 microprobes. Our results suggest that nanostructured SU-8 microprobes provide a flexible neural interface that enhances their long-term performance and stability.

\section{Methods \\ Fabrication of nanostructures on the surface of SU-8 microprobes}

To improve neuronal attachment and neurite outgrowth, we fabricated nanostructures on the surface of SU-8 microprobes, as shown in Fig. 1. Conventional photolithography and nanosphere lithography were used to fabricate the microprobe and nanostructures, respectively. The overall fabrication process is shown in Fig. 2. SU-8 2002 (MicroChem Corporation, Newton, MA, USA) was first spin-coated onto a glass wafer (Borofloat 33; Schott, Jena, Germany) for $30 \mathrm{~s}$ at $1500 \mathrm{rpm}$ (Fig. 2a). The wafer was baked for $10 \mathrm{~min}$ at $120{ }^{\circ} \mathrm{C}$; the resulting SU-8 film was $\sim 1.6 \mu$ m thick. Next, a 20 -nm-thick titanium layer and 150-nm-thick gold layer were deposited using an e-beam evaporator (SRN-200; Sorona Inc., South Korea). Ti functions as an adhesive layer between the SU-8 and Au layers. Next, AZ5214 photoresist (AZ 5214, Microchemicals GmbH, Germany) was spin-coated at $1550 \mathrm{rpm}$ to a target thickness of $\sim 2.2 \mu \mathrm{m}$ and baked for $45 \mathrm{~s}$ at $120{ }^{\circ} \mathrm{C}$. As a mask for electrode patterning, a $\sim 100 \mathrm{~nm}$ chromium (Cr) layer was deposited using an e-beam evaporator. AZ5214 was removed by sonication in acetone (JT Baker, Phillipsburg, NJ, USA), and $\mathrm{Ti} / \mathrm{Au}$ layers were patterned using a dry-etching system (FabStar; Top Technology Ltd., South Korea) for $90 \mathrm{~s}$ at $2 \mathrm{~m}$ Torr with $\mathrm{Cl}_{2}$ and Ar flow rates of 15 and $5 \mathrm{sccm}$, respectively. The inductively coupled plasma (ICP) source power and bias power with radio frequency (RF) were 500 and $250 \mathrm{~W}$, respectively. After removing the $\mathrm{Cr}$ mask layer using a CR-7 etchant (Cyantek, Fremont, CA, USA), the electrode patterns were fabricated as shown in Fig. 2b.

To fabricate nanostructures around the electrodes, a second SU-8 layer of identical thickness to the first was

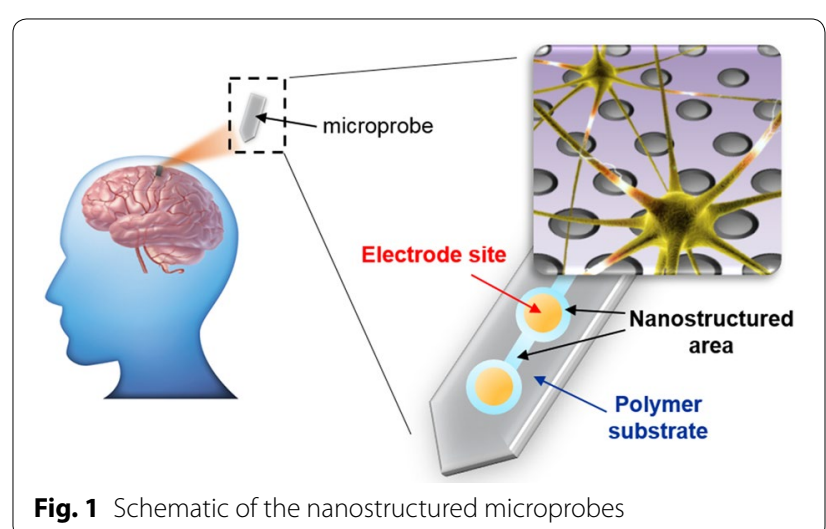


a

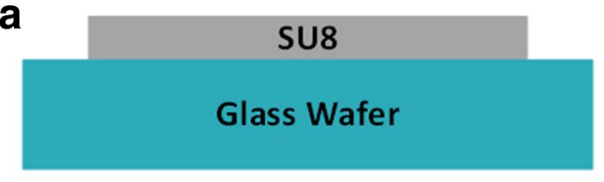

c

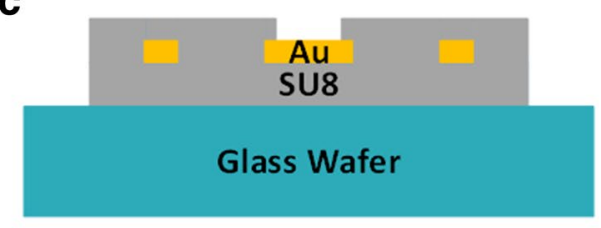

e

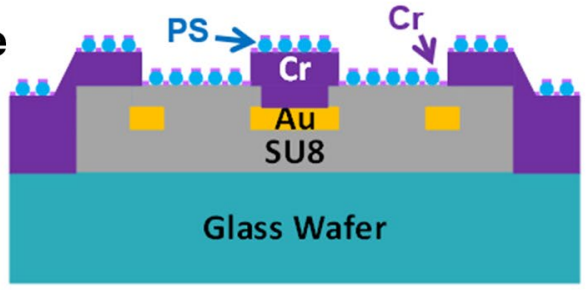

b

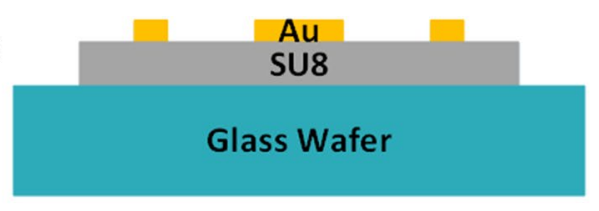

d
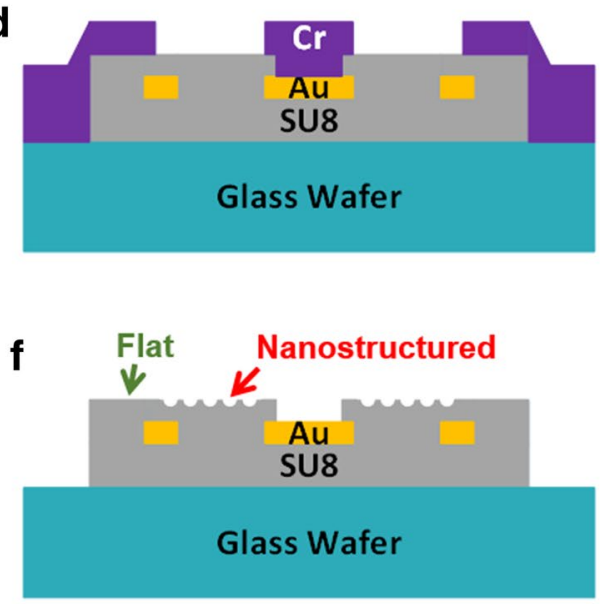

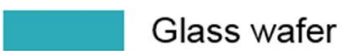

SU-8

Gold (Au)

\section{Chromium (Cr)}

Fig. 2 Fabrication of nanostructures on the microprobe surface: a negative photoresist coating on a glass wafer; b $\mathrm{Ti}$ and Au deposition using a Cr mask and inductively coupled plasma reactive-ion etching (ICP-RIE) of an Au layer as an electrode; c coating with an SU-8 passivation layer; d deposition of a Cr layer on the SU-8 microprobe; e self-assembly of polystyrene beads ( $300 \mathrm{~nm}$ diameter) and reduction of polystyrene bead diameter using RIE followed by deposition of a $30 \mathrm{~nm}$ Cr layer; and $\mathbf{f}$ removal of polystyrene beads by sonication in toluene for $1 \mathrm{~h}$, nanoscale etching of the SU-8 surface, and removal of the Cr mask using a Cr etchant

added using the same procedure (Fig. 2c). For the selective generation of nanostructures around the $\mathrm{Au}$ electrodes, a Cr layer was deposited and patterned by a lift-off process using a positive photoresist (AZ 5214) (Fig. 2d). For nanosphere lithography, the substrate with SU- 8 microprobes was cut into $18 \times 18 \mathrm{~mm}$ pieces, and coated with a monolayer of $0.3-\mu \mathrm{m}$-diameter polystyrene nanoparticles (PS NP suspension, 5.0\% coefficient of variation; Spherotech, Lake Forest, IL, USA) by transferring the PS monolayer to the substrate by the water-air interface [22]. The size of the PS nanobeads was reduced by oxygen plasma reactive-ion etching (RIE) (FabStar; Top Technology Ltd., South Korea) for 1 min with an $\mathrm{O}_{2}$ flow rate of $30 \mathrm{sccm}$ and power of $50 \mathrm{~W}$. A second 30-nmthick layer of $\mathrm{Cr}$ was deposited in the spaces among the PS nanobeads using an evaporator (Fig. 2e). The PS nanobeads were dissolved in toluene at $40{ }^{\circ} \mathrm{C}$ for $30 \mathrm{~min}$ with sonication, and nanostructures were generated by RIE with an $\mathrm{O}_{2}$ flow rate of $30 \mathrm{sccm}$ and power of $40 \mathrm{~W}$ for $4 \mathrm{~min}$. The Cr layer on SU-8 functions as an etch mask for the fabrication of nanostructures. Finally, the residual Cr layer (Fig. 2d, e) was removed using CR-7 etchant for $1 \mathrm{~min}$ and the substrate was cleaned in deionized water and disinfected in 100\% ethanol (Merck, Darmstadt, Germany) for $24 \mathrm{~h}$ in the dark. The substrate was washed three times in sterile distilled water and dried in a laminar flow hood.

\section{Characterization of the nanostructured surface}

A field-emission scanning electron microscope (FE-SEM) (S-4800; Hitachi, Tokyo, Japan) was used to observe the surface morphology of the nanostructured microprobes. An AFM (NX10; Park System Corp., South Korea) in non-contact mode in air at room temperature was used to measure the surface roughness and topography of the nanostructured surface.

\section{PC12 cell culture}

PC12 cells (CRL-1721; ATCC, Manassas, VA, USA) are extensively used in neurobiological and neurochemical studies [23, 24]. In this study, PC12 cells were used to investigate the responses of neurons to the nanostructured and non-nanostructured microprobes in vitro. PC12 cells were maintained in Roswell Park Memorial Institute (RPMI) 1640 medium with L-glutamine (Gibco, Grand Island, NY, USA) supplemented with 
10\% horse serum (Sigma-Aldrich, St. Louis, MO, USA), 5\% fetal bovine serum (FBS; Hyclone, Logan, UT, USA, and Thermo Fisher Scientific, Rockford, IL, USA) and 1\% antibiotic-antimycotic solution (penicillin 10,000 units/ $\mathrm{mL}$, streptomycin 10,000 $\mu \mathrm{g} / \mathrm{mL}$, Fungizone (amphotericin B) $25 \mu \mathrm{g} / \mathrm{mL}$; Gibco Life Technologies, Grand Island, NY, USA) at $37{ }^{\circ} \mathrm{C}$ in $5 \% \mathrm{CO}_{2}$ and subcultured at least twice weekly. For differentiation, PC12 cells were seeded onto plates at $2.5 \times 10^{5}$ cells $/ \mathrm{mL}$ and treated with $50 \mathrm{ng} / \mathrm{mL}$ nerve growth factor (NGF; BD Biosciences, Bedford, MA, USA) for 7 days. NGF-supplemented medium was changed every 2 days.

\section{Statistical analysis}

Neurites longer than $25 \mu \mathrm{m}$ (i.e., the average cell body diameter) were enumerated. Three areas were randomly selected on each substrate by scanning from top to bottom under a microscope. Experiments were conducted in triplicate. Comparisons were carried out using Student's $\mathrm{t}$ test. Results are presented as means \pm standard errors, and a value of $\mathrm{p}<0.001$ was considered to indicate statistical significance.

\section{Results and discussion}

Fabrication of SU-8-based microprobes

Nanostructured SU-8 microprobes were fabricated by conventional photolithography. Figure 3a shows the fabricated microprobes having four electrode sites. The total length of the microprobes was $4 \mathrm{~mm}$ (Fig. 3b, c). The shank width of the microprobe was $300 \mu \mathrm{m}$, the pitch of the recording sites was $200 \mu \mathrm{m}$, and the width of each opened electrode surface was $80 \mu \mathrm{m}$. The electrode sites were opened with a cloverleaf (Fig. 3c) and a star (Fig. 3d) shapes to induce cell adhesion and growth on the electrode. To prepare nanostructures in the desired area on the microprobe surface, the substrate was masked with Cr. Nanostructures were created around the electrode sites, as shown in Fig. 3d, e, which are not covered by the

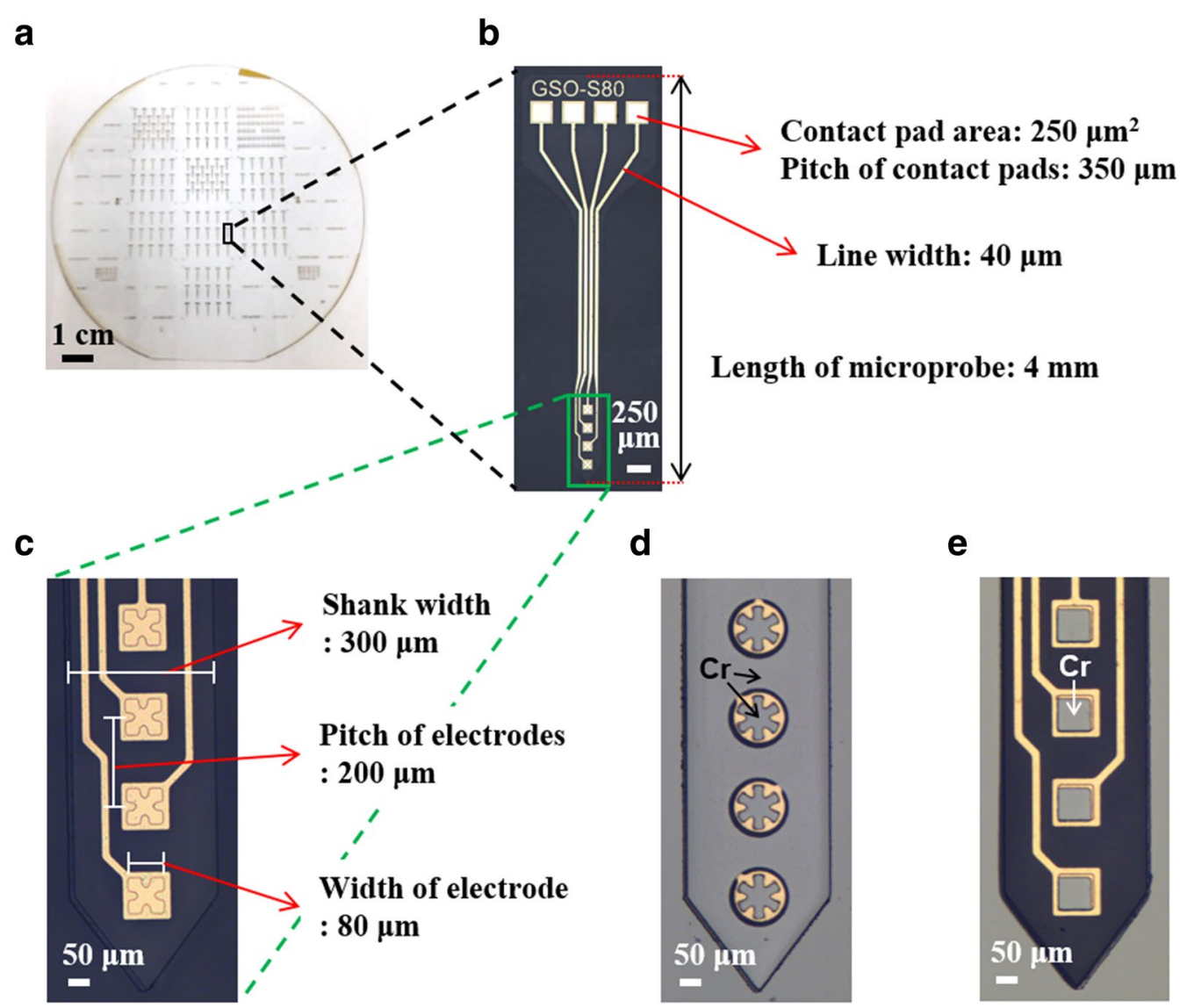

Fig. 3 Fabricated microprobe without or with a nanostructured surface. a Photograph of SU-8 microprobes on a 4-inch glass wafer. b Enlarged optical image of the microprobe in $(\mathbf{a})$ and $\mathbf{c}$ a representative optical image of gold electrode on the microprobe surface in (b). $\mathbf{d}$ Asterisk shape electrode and e square shape electrode of the microprobe masked with chromium (Cr) for nanostructuring 
Cr layer. As shown in Additional file 1: Figure S1, the area around the electrode sites was designed to have a $20 \mu \mathrm{m}$ wide guide path similar to the single cell size. The size of cultured neuronal cells has cell bodies with a diameter of $15-20 \mu \mathrm{m}[25,26]$. Cells can thus be attached to the surface of the nanostructured SU-8 microprobe compared to the non-nanostructured SU-8 microprobe, according to our previous study $[17,18]$. In addition, cells could be induced to lead to electrode sites by the pathway as they adhere to the microprobes. Therefore, there could be more neuronal cells on the electrode and increase the ability to record neuronal signals through electrodes.

\section{Characterization of surface nanostructures}

Figure 4 shows the results of surface morphologies on nanostructured SU-8 microprobes. An SEM image in Fig. 4a shows the surface of a gold electrode at the end of a microprobe. The microprobe surface was extensively covered with nanostructures, except around gold electrodes (Fig. 4b). Due to masking for the electrode site, there was no nanostructured topography on the gold electrode area (Fig. 4c). Figure 4d shows an enlarged SEM image of the nanostructures, which have an appearance similar to nano-holes. AFM was used to assess the depth of the nanostructures on the surface of SU-8 microprobes (Fig. 4e, f). The nanostructures exhibited similar appearances in AFM and SEM images. The nanoholes had a diameter and a depth of $\sim 200 \mathrm{~nm}$ (Fig. 4f); this is because filopodia have a diameter of $100-300 \mathrm{~nm}$; these structures play an important role in the outgrowth of neurites and serve as precursors to dendritic spines in neurons [27]. Therefore, we use this size to enhance neurite outgrowth on the nanostructured SU-8 microprobe. We successfully applied the nanostructuring technique to the surface of the SU-8 microprobe and obtained nanostructures that were consistent with our previous study [17].

\section{In vitro culture of PC12 cells on nanostructured SU-8 microprobes}

We assessed the neuronal development of PC12 cells on uncoated, nanostructured microprobes. Figure 5 shows neuronal outgrowth of PC12 cells on nanostructured and non-nanostructured microprobes. At 3 days after NGF treatment, PC12 cells were not attached to nonnanostructured microprobes and remained undifferentiated (Fig. 5a). In contrast, PC12 cells on nanostructured microprobes were not surface-bound on the 1-day in vitro culture (DIV1, 1 day after seeding), but differentiated 3 days after NGF treatment (Fig. 5b, c).

To compare neuronal development between nanostructured and non-nanostructured microprobes, the number of neurites extended per PC12 cell was determined in phase-contrast optical images. Figure $5 \mathrm{~d}$ shows the number of neurites per differentiated PC12 cell on non-nanostructured and nanostructured microprobes for 5 days after NGF treatment. Differentiation of PC12 cells was significantly greater on nanostructured than on non-nanostructured microprobes ( $\mathrm{p}<0.001)$. In contrast, few PC12 cells were attached to non-nanostructured microprobes, and those that did attach were in close proximity to each other (Fig. 5a). Because PC12 cells differentiate as they attach to the surface, similar to our previous study, they showed low neurite outgrowth over time at 5 days after NGF treatment [18]. It is interesting to note that the length of PC12 neurite extension on nanostructured microprobes is similar to that on rat tail collagen type I coated culture dishes [28]. As shown in Additional file 1: Figure S2, PC12 cells extended to the longest neurite length for about $150 \mu \mathrm{m} 10$ days after NGF treatment. Surface attachment and growth by PC12 cells typically require an adhesive coating (e.g., PLL), but nanostructured microprobes enhance cell adhesion and differentiation without the requirement for a surface coating. Nanostructures are permanent; in contrast, the effect on cell adhesion of a coating of adhesion molecules is limited by the inevitable degradation over time [29]. Therefore, nanostructured microprobes support neuronal growth for longer periods than those with conventional surface coatings.

Further studies should determine whether nanostructured microprobes support neuronal growth over the long term in comparison with microprobes coated with cell-adhesion molecules. After microprobe implantation, the foreign-body reaction and inflammatory response should be evaluated to validate the utility of nanostructured microprobes in vivo. Nanostructured microprobes show promise for implantable brain-machine interfaces that can remain in situ for long periods.

\section{Conclusion}

In this study, nanostructured SU-8 microprobes were developed to enhance neuronal growth with the aim of facilitating long-term recording of neuronal signals. SU-8 microprobes were produced by conventional photolithography and nanostructures of $\sim 200 \mathrm{~nm}$ diameter and depth were generated on their surfaces. Neuronal development on nanostructured and non-nanostructured microprobes was characterized using PC12 neuron-like cells. PC12 cells on nanostructured microprobes showed significantly increased attachment and neurite outgrowth compared with those on non-nanostructured microprobes. This improved neuronal development did not require an adhesive coating (e.g., PLL) and so the nanostructured 

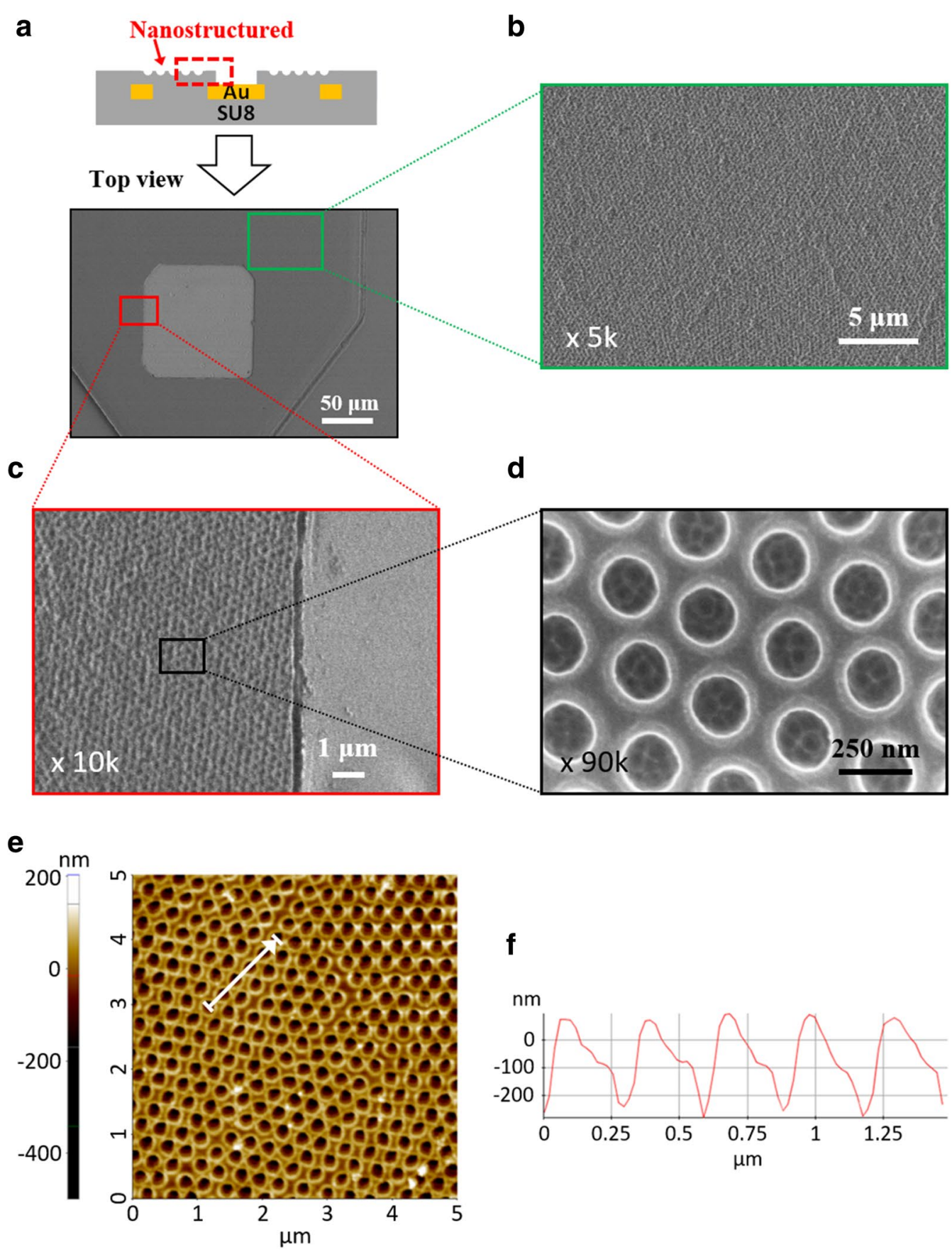

Fig. 4 Nanostructured area of a microprobe. a Schematic of a nanostructured area on the microprobe surface, and SEM image of the top of a microprobe showing a gold electrode. SEM images of the boxed areas in (a); $\mathbf{b}$ nanostructured area; $\mathbf{c}$ boundary between a nanostructured area and a gold electrode; $\mathbf{d}$ enlargement of the boxed area in (c); e AFM image $(5 \times 5 \mu \mathrm{m})$ of a nanostructured microprobe surface; and $\mathbf{f}$ line profile of the white arrow in (e). Nano-holes are $\sim 200 \mathrm{~nm}$ in both diameter and depth 
a

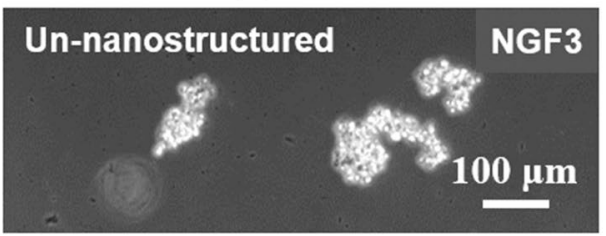

b

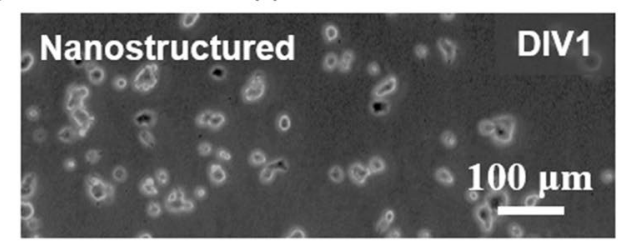

C

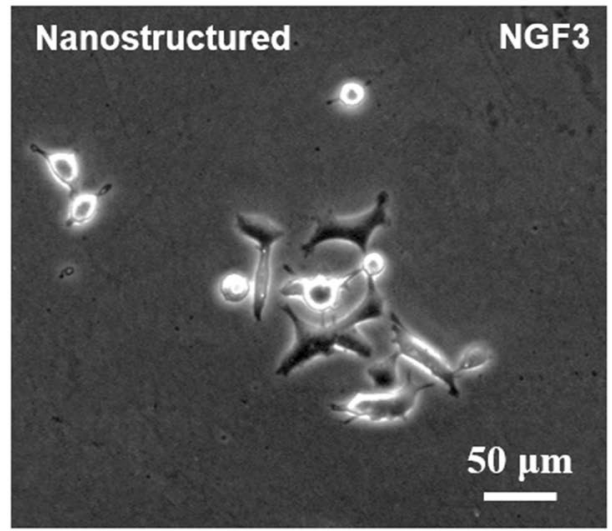

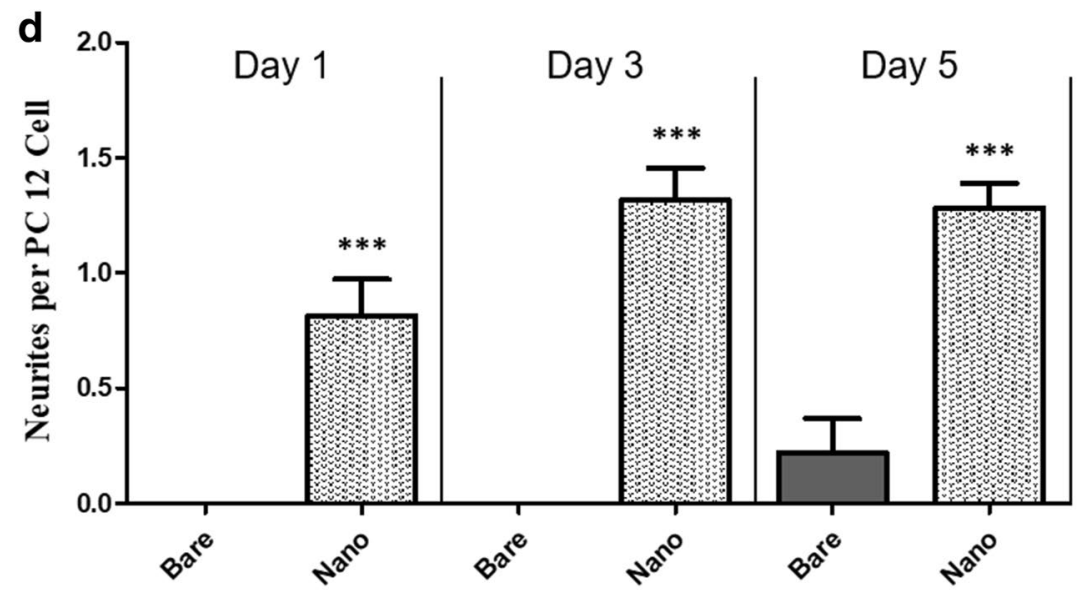

Time (Days)

Fig. 5 Phase-contrast optical images of rat PC12 cells cultured on nanostructured and non-nanostructured microprobes. a At 3 days after NGF treatment, PC12 cells were not attached or differentiated on non-nanostructured microprobes (magnification, $\times 100)$. PC12 cells on a nanostructured microprobe b 1-day in vitro culture (DIV1), before the addition of NGF (magnification, $\times 100$ ), and $\mathbf{c} 3$ days after NGF treatment (magnification, $\times 200)$. Cell adhesion and differentiation have been improved for nanostructured microprobes compared to non-nanostructured microprobes. d Number of neurites per differentiated PC12 cell on non-nanostructured and nanostructured microprobes at 5 days after NGF treatment (Student's $t$ test; mean $\pm \mathrm{SE}, \mathrm{n}=9$, $\left.{ }^{* * *} \mathrm{p}<0.001\right)$

microprobes show promise for use in implantable devices that support neurons, for example, implantable brainmachine interfaces.

\section{Additional file}

Additional file 1. Design of SU-8 microprobe for nanostructured area around electrode site.

Additional file 2. Biocompatibility of PC12 cells on nanostructured SU-8 microprobe.

\section{Authors' contributions}

EK carried out the experiment and drafted the manuscript. EK, JK and HC conceived of the study. All authors read and approved the final manuscript.

\section{Author details}

${ }^{1}$ DGIST-ETH Microrobotics Research Center (DEMRC), Daegu Gyeongbuk Institute of Science and Technology (DGIST), Daegu 42988, South Korea. 2 Department of Robotics Engineering, Daegu Gyeongbuk Institute of Science and Technology (DGIST), Daegu 42988, South Korea.

\section{Acknowledgements}

We thank the Center for Core Research Facilities of DGIST for technical support. The authors are also grateful to Sangwon Kim and Jongmoon Jang (DGIST) for their help with the SEM inspection. 


\section{Competing interests}

The authors declare that they have no competing interests.

\section{Ethics approval and consent to participate}

Not applicable.

\section{Funding}

Funding for this research was provided by the Global Research Laboratory from the National Research Foundation of Korea (NRF), funded by the Ministry of Science and ICT (No. NRF 2017K1A1A2013237), Korea Evaluation Institute of Industrial Technology (KEIT) funded by the Ministry of Trade, Industry \& Energy (No. 10052980), and by DGIST MIREBraiN Project.

\section{Publisher's Note}

Springer Nature remains neutral with regard to jurisdictional claims in published maps and institutional affiliations.

Received: 31 August 2017 Accepted: 1 December 2017 Published online: 06 December 2017

\section{References}

1. Abbott A (2006) Neuroprosthetics: in search of the sixth sense. Nature 442(7099):125-127

2. Moxon K, Hallman S, Sundarakrishnan A, Wheatley M, Nissanov J, Barbee K (2009) Long-term recordings of multiple, single-neurons for clinical applications: the emerging role of the bioactive microelectrode. Materials 2(4):1762

3. Polikov VS, Tresco PA, Reichert WM (2005) Response of brain tissue to chronically implanted neural electrodes. J Neurosci Methods 148(1):1-18

4. He W, McConnell GC, Bellamkonda RV (2006) Nanoscale laminin coating modulates cortical scarring response around implanted silicon microelectrode arrays. J Neural Eng 3(4):316

5. He W, Bellamkonda RV (2005) Nanoscale neuro-integrative coatings for neural implants. Biomaterials 26(16):2983-2990

6. Azemi E, Stauffer WR, Gostock MS, Lagenaur CF, Cui XT (2008) Surface immobilization of neural adhesion molecule 11 for improving the biocompatibility of chronic neural probes: in vitro characterization. Acta Biomater 4(5):1208-1217

7. Jun SB, Hynd MR, Dowell-Mesfin NM, Al-Kofahi Y, Roysam B, Shain W Kim SJ (2008) Modulation of cultured neural networks using neurotrophin release from hydrogel-coated microelectrode arrays. J Neural Eng 5(2):203

8. Zhang L, Webster TJ (2009) Nanotechnology and nanomaterials: promises for improved tissue regeneration. Nano Today 4(1):66-80

9. Park J, Moon D, Kim J, Lee J (2016) Cell adhesion and growth on the anodized aluminum oxide membrane. J Biomed Nanotechnol 12(3):575-580

10. Piret G, Perez M-T, Prinz CN (2013) Neurite outgrowth and synaptophysin expression of postnatal cns neurons on gap nanowire arrays in long-term retinal cell culture. Biomaterials 34(4):875-887

11. Robinson JT, Jorgolli M, Shalek AK, Yoon M-H, Gertner RS, Park H (2012) Vertical nanowire electrode arrays as a scalable platform for intracellular interfacing to neuronal circuits. Nat Nanotechnol 7(3):180-184

12. Cesca F, Limongi T, Accardo A, Rocchi A, Orlando M, Shalabaeva $V$, Di Fabrizio E, Benfenati F (2014) Fabrication of biocompatible free-standing nanopatterned films for primary neuronal cultures. RSC Adv 4(86):45696-45702

13. Chapman CA, Wang L, Chen H, Garrison J, Lein PJ, Seker E (2017) Nanoporous gold biointerfaces: modifying nanostructure to control neural cell coverage and enhance electrophysiological recording performance. Adv Funct Mater 27(3):1604631

14. Chapman CA, Chen H, Stamou M, Biener J, Biener MM, Lein PJ, Seker E (2015) Nanoporous gold as a neural interface coating: effects of topography, surface chemistry, and feature size. ACS Appl Mater Interfaces 7(13):7093-7100

15. Moxon KA, Kalkhoran NM, Markert M, Sambito MA, McKenzie J, Webster JT (2004) Nanostructured surface modification of ceramic-based microelectrodes to enhance biocompatibility for a direct brain-machine interface. IEEE Trans Biomed Eng 51(6):881-889

16. Moxon K, Hallman S, Aslani A, Kalkhoran N, Lelkes P (2007) Bioactive properties of nanostructured porous silicon for enhancing electrode to neuron interfaces. J Biomater Sci Polym Ed 18(10):1263-1281

17. Kim E, Yoo S-J, Moon C, Nelson BJ, Choi H (2015) Su-8-based nanoporous substrate for migration of neuronal cells. Microelectron Eng 141:173-177

18. Kim E, Yoo S-J, Kim E, Kwon T-H, Zhang L, Moon C, Choi H (2016) Nanopatterned su-8 surface using nanosphere-lithography for enhanced neuronal cell growth. Nanotechnology 27(17):175303

19. Nemani KV, Moodie KL, Brennick JB, Su A, Gimi B (2013) In vitro and in vivo evaluation of SU-8 biocompatibility. Mater Sci Eng 33(7):4453-4459

20. Cho S-H, Lu HM, Cauller L, Romero-Ortega MI, Lee J-B, Hughes GA (2008) Biocompatible SU-8-based microprobes for recording neural spike signals from regenerated peripheral nerve fibers. IEEE Sens J 8(11):1830-1836

21. Jeong G, Park J, Lee K, Jang J, Lee C, Kang H, Yang C, Suh S (2010) Fabrication of low-cost mold and nanoimprint lithography using polystyrene nanosphere. Microelectron Eng 87(1):51-55

22. Ho C-C, Chen P-Y, Lin K-H, Juan W-T, Lee W-L (2011) Fabrication of monolayer of polymer/nanospheres hybrid at a water-air interface. ACS Appl Mater Interfaces 3(2):204-208

23. Fujita K, Guroff G, Yavin E, Goping G, Orenberg R, Lazarovici P (1990) Preparation of affinity-purified, biotinylated tetanus toxin, and characterization and localization of cell surface binding sites on nerve growth factor-treated pc12 cells. Neurochem Res 15(4):373-383

24. Greene LA, Tischler AS (1976) Establishment of a noradrenergic clonal line of rat adrenal pheochromocytoma cells which respond to nerve growth factor. Proc Natl Acad Sci 73(7):2424-2428

25. Offenhäusser A, Sprössler C, Matsuzawa M, Knoll W (1997) Field-effect transistor array for monitoring electrical activity from mammalian neurons in culture. Biosens Bioelectron 12(8):819-826

26. Reier PJ, Perlow MJ, Guth L (1983) Development of embryonic spinal cord transplants in the rat. Dev Brain Res 10(2):201-219

27. Mattila PK, Lappalainen P (2008) Filopodia: molecular architecture and cellular functions. Nat Rev Mol Cell Biol 9(6):446-454

28. Attiah DG, Kopher RA, Desai TA (2003) Characterization of PC12 cell proliferation and differentiation-stimulated by ECM adhesion proteins and neurotrophic factors. J Mater Sci 14(11):1005-1009

29. Mingyu C, Kai G, Jiamou L, Yandao G, Nanming Z, Xiufang Z (2004) Surface modification and characterization of chitosan film blended with poly-L-lysine. J Biomater Appl 19(1):59-75 